\title{
Attacking latent HIV: towards eradication of HIV infection DM Margolis*
}

Address: University of North Carolina at Chapel Hill, NC, USA

* Corresponding author

from 2006 International Meeting of The Institute of Human Virology Baltimore, USA. 17-21 November, 2006

Published: 2I December 2006

Retrovirology 2006, 3(SuppI I):SI0 doi:I0.II86/I742-4690-3-SI-SI0

(C) 2006 Margolis; licensee BioMed Central Ltd.

Targeting latent infection within resting CD4+ T cells might lead to better control, and perhaps eradication of HIV infection. Histone deacetylase 1 (HDAC1) plays a central role maintaining HIV latency. HDAC inhibition augments HIV promoter and viral expression without global $\mathrm{T}$ cell activation or increased de novo HIV infection. We tested the ability of the HDAC inhibitor valproic acid (VPA) to deplete replication-competent HIV in vivo within the resting CD4+ T cell pool in stably aviremic, patients on standard combination antiretroviral therapy (ART).

We intensified therapy by the addition of enfuvirtide (T20) agent prior to VPA treatment. Four volunteers with plasma HIV RNA $<50$ copies $/ \mathrm{ml}$ for mean of $>46$ months added T-20 for 4-6 weeks, and then VPA to their therapy for 3 additional months. The frequency of replicationcompetent HIV in resting CD4+ T cells was enumerated by limiting-dilution culture of maximally activated resting CD4+ T cells.

Repeated assays within patients did not demonstrate a decline of resting cell infection on ART. A significant decline (mean reduction 75\%; range 68\% to >84\%) occurred in three of four patients after treatment with T-20 and VPA.

Initial data in ongoing studies of ART and VPA without intensification demonstrate depletion of latent infection in some patients. Laboratory studies of other HDAC inhibitors and of other mechanisms that contribute to persistent, quiescent proviral infection suggest that clinical strategies may eventually be developed that can induce expression and clearance of HIV, and avoid the need for lifelong ART in some patients. 